\title{
Increased Prevalence of Systemic Lupus Erythematosus Comorbidity in Patients With Psoriatic Arthritis: A Population-based Case-control Study
}

\author{
Danielle Korkus ${ }^{1}$, Tal Gazitt ${ }^{2}$ (D), Arnon Dov Cohen ${ }^{4}$, Ilan Feldhamer ${ }^{3}$, Idit Lavi ${ }^{5}$, Amir Haddad², \\ Sari Greenberg-Dotan ${ }^{3}$, Erez Batat ${ }^{3}$, and Devy Zisman ${ }^{6}$
}

\begin{abstract}
Objective. To assess the prevalence of systemic lupus erythematosus (SLE) in a psoriatic arthritis (PsA) cohort and to compare it to the general population using the database of a large healthcare provider.

Methods. We analyzed the database of a PsA cohort (2002-2017), matched for age and sex, with randomly selected controls for demographics, clinical and laboratory manifestations, and dispensed medications. Statistical analysis used $t$ test and chi-square test as appropriate. In the PsA group, incidence density sampling was performed matching PsA patients without SLE as controls to each case of PsA with SLE by age and follow-up time. Univariable and multivariable conditional logistic regression analyses were used to assess factors affecting SLE development.

Results. The PsA and control groups consisted of 4836 and 24,180 subjects, respectively, with a median age of $56 \pm 15$ years, and of whom $53.8 \%$ were female. Eighteen patients (0.37\%) in the PsA group and 36 patients $(0.15 \%)$ in the control group were diagnosed with SLE $(P=0.001)$. SLE patients without PsA had higher anti-dsDNA and anticardiolipin antibodies. The usage of drugs with known potential to induce SLE was higher in the PsA than in the control group. Older age at PsA diagnosis, shorter PsA duration, and statin treatment were associated with SLE in PsA patients.

Conclusion. A 2.3-fold increase in the prevalence of SLE in PsA relative to the control group was found. Risk factors for SLE development included older age at PsA diagnosis, shorter PsA duration, and statin treatment. The association between PSA and SLE may affect treatment choices and medication development.
\end{abstract}

Key Indexing Terms: comorbidities, psoriatic arthritis, spondyloarthropathy, systemic lupus erythematosus

Psoriatic arthritis (PsA) is an inflammatory musculoskeletal disease with immune-mediated features. The disease is classified as part of the spondyloarthritides. PsA occurs in 25\% of individuals with psoriasis, and up to $1 \%$ of the general population ${ }^{1,2}$. Patients with psoriasis and PsA can develop a variety of comorbidities including diabetes, hypertension, cardiovascular diseases, and depression. Patients with PsA are more affected than those

${ }^{I} D$. Korkus, MD, The Ruth and Bruce Rappaport Faculty of Medicine,

Technion, Haifa; ${ }^{2}$ T. Gazitt, MD, MSc, Amir Haddad, MD, Rheumatology

Unit, Carmel Medical Center, Haifa; ${ }^{3}$ I. Feldhamer, MA, S. Greenberg-Dotan,

PhD, E. Batat, MBA, Chief Physician's Office, Central Headquarters, Clalit

Health Services, Tel Aviv; ${ }^{4}$ A. Dov Cohen, PhD, MD, MPH, Chief Physician's

Office, Central Headquarters, Clalit Health Services, Tel Aviv, Israel, and

Siaal Research Center for Family Medicine and Primary Care, Faculty of

Health Sciences, Ben-Gurion University of the Negev, Beer-Sheba; ${ }^{5}$ I. Lavi,

MPH, MA, Department of Community Medicine and Epidemiology, Carmel

Medical Center, Haifa; ${ }^{6} D$. Zisman, MD, The Ruth and Bruce Rappaport

Faculty of Medicine, Technion, and Department of Community Medicine and

Epidemiology, Carmel Medical Center, Haifa, Israel.

T. Gazitt and A.D. Cohen contributed equally to this manuscript.

Address correspondence to Dr. D. Zisman, Rheumatology Unit,

Carmel Medical Center, 7 Michal Street, Haifa 34362, Israel.

Email:devyzisman@gmail.com.

Full Release Article. For details see Reprints and Permissions at jrheum.org.

Accepted for publication May 6, 2020. with isolated psoriasis, and disease severity also increases the prevalence of comorbidities ${ }^{3}$. Comorbidities, in turn, may influence the therapeutic regimen and affect treatment outcomes.

Systemic lupus erythematosus (SLE) is an autoimmune disease most common in women of childbearing age. In the United States, the prevalence varies from 20 to 50 per 100,000 women. The disease affects many different organs and the clinical manifestations include the musculoskeletal, cutaneous, renal, central nervous hematologic, cardiac, and gastrointestinal systems ${ }^{1}$. The presence of antinuclear antibodies (ANA) is one of the hallmarks of SLE ${ }^{4}$. Several studies report a higher prevalence of ANA positivity in patients with psoriasis and $\mathrm{PsA}^{5,67}$. Previous studies also report a coexistence of psoriasis and SLE ${ }^{8,9}$, but unlike psoriasis, the coexistence of PsA and SLE has only been reported in several case reports ${ }^{9,10}$. Our study objectives were to assess the prevalence of SLE in a PsA patient cohort and to compare it to the general population.

\section{MATERIALS AND METHODS}

Study population. The subjects and information used in this study derive from the Clalit Health Services (CHS) database. The CHS serves approximately $52 \%$ of the Israeli population ( 4.3 million people). The CHS database includes information updated continuously from pharmaceutical, medical, and administrative operating systems. Disease codes are registered according to the International Classification of Diseases, Ninth Revision 
(ICD-9) and medications dispensed are coded according to the Anatomical Therapeutic Chemical classification. The database includes information on all medications dispensed including the date, dose, and mode of administration. The database was designed for the purposes of administrative and clinical management and is available for use in epidemiological studies. The PsA patient cohort was validated and described in detail in previous studies ${ }^{11,12}$. For each patient with PsA found in the registry from January 1, 2002 to December 31, 2017, 5 age- and sex-matched subjects who had no history of psoriasis, PsA, rheumatoid arthritis, or ankylosing spondylitis were chosen as a control group from the entire CHS database. In both groups, patients with a code diagnosis of SLE were identified. The diagnosis of SLE was then validated based on clinical and laboratory manifestations of the disease using the Systemic Lupus International Collaborating Clinics (SLICC) classification criteria ${ }^{4}$ by manually exploring the database, including rheumatologist and other specialist clinic visit notes and reviewing hospital discharge summaries and laboratory results.

Demographic data included in this study from the CHS database included age, sex, ethnicity (Jewish/Arab), and socioeconomic status (SES) at enrolment, the latter defined by the categories of low, medium, or high, that correlate highly with the Israel Central Bureau of Statistics categories of SES status and data on BMI. In addition, dispensed medications such as glucocorticosteroids, nonsteroidal antiinflammatory drugs (NSAID), conventional disease-modifying antirheumatic drugs (cDMARD), biological disease-modifying antirheumatic drugs (bDMARD), mycophenolate mofetil, cyclophosphamide, and medications with known potential to induce SLE ${ }^{13,14}$, as well as phototherapy [broadband UVA/UVB, narrow band UVB, and psoralen UVA (PUVA)], were analyzed.

In this study, ANA was measured by immunofluorescence (IF) read manually by a laboratory technician and by multiplex bead assay. The cutoff dilution was set at 1:160. Anti-dsDNA positivity was validated by Crithidia assay (AESKUSLIDES nDNA Crithidia Lucilia Test).

The study was approved by the Institutional Review Board of Carmel Medical Center (CMC-0014-14). The requirement for individual patient consent forms was waived due to the retrospective, observational nature of the study.

Statistical analysis. Descriptive analysis of the study population comparing PsA patients with and without coexisting SLE, and between this PsA patient group and the control group, was performed using chi-square test and Student $t$-test, as appropriate. The standardized incidence ratio (SIR) comparing the prevalence of SLE among patients with PsA compared to the control group was calculated, adjusted for age and sex. Posthoc analysis was used to develop a predictive model for SLE development among PsA patients by using incidence density sampling (SAS/ACCESS version 9.4, released 2013, SAS Institute Inc.) in which we attempted to match 10 comparator PsA female patients without coexisting SLE as controls to each case of PsA with coexisting SLE by date of birth and follow-up time, which was defined as the date of SLE diagnosis. The association between SLE occurrence, adjusted to PsA duration or age at PsA diagnosis, was analyzed using 2 separate multivariable conditional logistic regression models: model I which included PsA duration; and Model II which included age at PsA diagnosis. A third model, Model III, in which we included the entire study population (i.e., the control group and the PsA patient group) and analyzed the association of age, sex, PsA diagnosis, ethnicity, and possible medications used prior to SLE diagnosis, was used to calculate the risk of SLE development. OR and 95\% CI were calculated in order to estimate the association between the variables and SLE events. All data were analyzed using SPSS, version 24 (IBM SPSS Statistics for Windows, version 24.0). All tests were 2-sided; $P$ values of $<0.05$ were considered statistically significant.

\section{RESULTS}

The PsA study group consisted of 4836 subjects, with a median age of $56 \pm 15$ years, 2603 (53.8\%) of whom were females. The control group consisted of 24,180 subjects matched for age and sex. In comparison to the control group, the PsA study group had a lower SES (32.2\% vs $40.6 \%, P<0.0001)$, higher percentage of smokers ( $42.5 \%$ vs $38.3 \%, P<0.0001)$, higher BMI $(28.65 \pm 5.8$ vs $27.5 \pm 5.4, P<0.0001)$, and had a higher percentage of Jewish patients $(87.3 \%$ vs $81.5 \%, P<0.0001)$. Comparing comorbidities between the study and control groups showed that the study group had a statistically significantly higher incidence of hyperlipidemia (64.6\% vs 55.6\%, $P<0.0001)$, hypertension $(42.1 \%$ vs $34.7 \%, P<0.0001)$, diabetes mellitus $(28.0 \%$ vs $22.1 \%$, $P<0.0001)$, malignancy (20.3\% vs $11.2 \%, P<0.0001)$, osteoporosis (12.9\% vs $9.8 \%, P<0.0001)$, obesity $(38.3 \%$ vs $28.1 \%$, $P<0.0001)$, congestive heart failure ( $4.5 \%$ vs $3.2 \%, P<0.0001)$, ischemic heart disease $(15.8 \%$ vs $12.4 \%, P<0.0001)$, and cerebrovascular accident (6.3\% vs $5.4 \%, P<0.0001$; Table 1$)$.

Overall, out of this PsA cohort, 18 patients (0.37\%) met SLE classification criteria versus 36 patients $(0.15 \%)$ in the control group, with $P=0.001$ (Table 1). The calculated SIR comparing the prevalence of SLE among patients with PsA to the control group reached 2.36 when adjusted for age, and 2.81 when adjusted for age and sex. Ten patients were diagnosed with SLE before PsA diagnosis with a mean of $15.62 \pm 17.48$ years and a median of 10.46 years. Eight patients were diagnosed with PsA before SLE diagnosis with a mean of $4.84 \pm 6.79$ years and a median of 2.45 years.

SLE patients without coexisting PsA were more likely to be positive for anti-dsDNA $(91.7 \%$ vs $66.7 \%, P=0.047)$ and anticardiolipin antibodies ( $47.2 \%$ vs $16.7 \%, P=0.038)$. Antihistone antibodies were found in 6 of $36(16.7 \%)$ control patients and in the same percentage of PsA patients, 3 of 18 (16.7\%). No other significant differences were observed between the 2 groups in terms of clinical and laboratory manifestations of SLE (Table 2). PsA patients with concomitant SLE versus PsA patients without SLE were more often female ( $100 \%$ vs $53.7 \%, P<0.0001$ ), more likely to have osteoporosis ( $38.9 \%$ vs $12.8 \%, P=0.005)$, and more likely to be treated with beta blockers $(27.8 \%$ vs $9.8 \%, P=$ 0.027; Table 3). Usage of medications with known potential to induce SLE prior to diagnosis of SLE was higher in the study group of PsA patients with concomitant SLE (11 of 18 patients) than in the control group. Possible culprit medications associated with later onset of SLE included beta blockers $(0.4 \%$ in the PsA cohort vs $0.004 \%$ in the control group, $P=0.07)$; statins ( $0.15 \%$ vs $0.02 \%, P=0.001)$; and anti-tumor necrosis factor- $\alpha$ (anti-TNF- $\alpha$ ) agents ( $0.4 \%$ vs $0.0 \%, P=0.028$; Table 1$)$. None of the 18 patients in the PsA group were treated with phototherapy prior to SLE diagnosis. No difference in clinical manifestations of SLE was observed between patients treated with medications known to induce SLE relative to SLE patients not on such medications (Table 3). Out of the PsA group of 4836 patients, 4062 were examined for ANA positivity, with 1189 (29.3\%) having a positive test result at some timepoint over their follow-up period.

Using 2 different multivariable conditional logistic regression models, we analyzed potential risk factors for the future development of SLE among patients with PsA by matching 131 patients with PsA to the 18 PsA patients who developed SLE (Table 4). In model I, where PsA duration was analyzed, 
Table 1. Characteristics of the study population.

\begin{tabular}{lccc}
\hline & Controls & PsA & $P$ \\
\hline No. patients & 24,180 & 4836 & - \\
SLE cases & $36(0.15)$ & $18(0.37)$ & 0.001 \\
Age, yrs, mean \pm SD & $56.25 \pm 15.26$ & $56.31 \pm 15.24$ & $\mathrm{NS}$ \\
Sex, female & $13,015(53.8)$ & $2603(53.8)$ & $\mathrm{NS}$ \\
SES & & & \\
1 & $9082(40.6)$ & $1552(32.2)$ & $<0.0001$ \\
2 & $8336(37.3)$ & $1958(40.7)$ & $<0.0001$ \\
3 & $4956(22.2)$ & $1306(27.1)$ & $<0.0001$ \\
Smokers & $9220(38.3)$ & $2051(42.5)$ & $<0.0001$ \\
Ethnicity & & & \\
$\quad$ Arab & $4369(18.5)$ & $612(12.7)$ & $<0.0001$ \\
Jewish & $19,296(81.5)$ & $4224(87.3)$ & \\
BMI, mean \pm SD & $27.47 \pm 5.44$ & $28.65 \pm 5.80$ & $<0.0001$ \\
Comorbidity & & & \\
Hyperlipidemia & $13,541(56)$ & $3125(64.6)$ & $<0.0001$ \\
Hypertension & $8383(34.7)$ & $2035(42.1)$ & $<0.0001$ \\
DM & $5339(22.1)$ & $1353(28)$ & $<0.0001$ \\
Malignancy & $2709(11.2)$ & $980(20.3)$ & $<0.0001$ \\
Osteoporosis & $2376(9.8)$ & $623(12.9)$ & $<0.0001$ \\
Obesity & $6790(28.1)$ & $1851(38.3)$ & $<0.0001$ \\
CHF & $758(3.2)$ & $218(4.5)$ & $<0.0001$ \\
IHD & $3000(12.4)$ & $766(15.8)$ & $<0.0001$ \\
CVA & $1312(5.4)$ & $304(6.3)$ & 0.017 \\
cDMARD & $578(2.4)$ & $4090(84.6)$ & $<0.0001$ \\
bDMARD & & & \\
$\quad$ Anti-TNF- $\alpha$ & $97(0.4)$ & $1779(36.8)$ & $<0.0001$ \\
Possible SLE-inducing & & & \\
medication used prior & & & NS \\
to SLE diagnosis & & & \\
Statins & $5(0.02)$ & $7(0.15)$ & 0.001 \\
PPI & $12(0.05)$ & $5(0.1)$ & $\mathrm{NS}$ \\
Beta blockers & $1(0.004)$ & $2(0.04)$ & 0.07 \\
ACEi & $7(0.03)$ & $1(0.02)$ & $\mathrm{NS}$ \\
Thiazides & $7(0.03)$ & $1(0.02)$ & \\
Anti-TNF- $\alpha$ & $0(0.0)$ & $2(0.04)$ & \\
\hline
\end{tabular}

Values are n (\%) unless otherwise specified. The percentages reported were calculated from the actual number, not including missing data. ACEi: angiotensin-converting enzyme inhibitor; anti-TNF- $\alpha$ : anti-tumor necrosis factor- $\alpha$; bDMARD: biological disease-modifying antirheumatic drug; cDMARD: conventional disease-modifying antirheumatic drug; CHF: congestive heart failure; CVA: cerebrovascular accident; DM: diabetes mellitus; IHD: ischemic heart disease; NS: not significant; PPI: proton pump inhibitor; PsA: psoriatic arthritis; SES: socioeconomic status; SLE: systemic lupus erythematosus.

we found an inverse relationship between PsA duration and SLE development such that patients with long-standing PsA were at lower risk of SLE development (OR 0.82, 95\% CI 0.69-0.98, $P=0.03$ ); in model II, where age at PsA diagnosis was analyzed, we found a direct relationship between age at PsA onset and risk of developing SLE, such that patients with later onset of PsA were at higher risk of SLE development relative to patients with early-age onset of PsA (OR 1.25, 95\% CI 1.06-1.48, $P=0.008$ ). Statin therapy was also found to increase the risk of SLE onset among PsA patients (Table $1 ; P<0.0001$ ). In model III, in which we included the entire study population consisting of the control
Table 2. Comparison between SLE patients in the 2 groups.

\begin{tabular}{|c|c|c|c|}
\hline & Controls & PsA & $P$ \\
\hline No. patients & 36 & 18 & - \\
\hline Age, yrs, mean \pm SD & $53.67 \pm 12.88$ & $55.61 \pm 15.99$ & NS \\
\hline Sex, female & $32(88.9)$ & $18(100)$ & NS \\
\hline \multicolumn{4}{|l|}{ SES } \\
\hline 1 & $14(38.9)$ & $8(44.4)$ & \multirow[t]{3}{*}{ NS } \\
\hline 2 & $15(41.7)$ & $5(27.8)$ & \\
\hline 3 & $7(19.4)$ & $5(27.8)$ & \\
\hline Smoker & $14(38.9)$ & $7(38.9)$ & NS \\
\hline \multicolumn{4}{|l|}{ Ethnicity } \\
\hline Arab & $7(19.4)$ & $3(16.7)$ & \multirow[t]{2}{*}{ NS } \\
\hline Jewish & $29(80.6)$ & $15(83.3)$ & \\
\hline \multicolumn{4}{|l|}{ Comorbidity } \\
\hline Hyperlipidemia & $16(44.4)$ & $9(50)$ & NS \\
\hline Hypertension & $17(47.2)$ & $8(44.4)$ & NS \\
\hline $\mathrm{DM}$ & $8(22.2)$ & $4(22.2)$ & NS \\
\hline Malignancy & $3(8.3)$ & $5(27.8)$ & NS \\
\hline CVD & $10(27.8)$ & $1(5.6)$ & NS \\
\hline Osteoporosis & $7(19.4)$ & $4(22.2)$ & NS \\
\hline Obesity & $7(19.4 .5)$ & $2(11.1)$ & NS \\
\hline \multicolumn{4}{|l|}{ SLE clinical manifestations } \\
\hline Skin & $23(63.9)$ & $12(66.7)$ & NS \\
\hline Oral ulcers & $9(25)$ & $6(33.3)$ & NS \\
\hline Alopecia & $9(25)$ & $8(44.4)$ & NS \\
\hline Arthritis & $28(77.8)$ & $15(83.3)$ & NS \\
\hline Renal & $12(33.3)$ & $4(22.2)$ & NS \\
\hline Neurologic & $5(13.9)$ & $0(0)$ & NS \\
\hline Hemolytic anemia & $1(2.8)$ & $3(16.7)$ & NS \\
\hline Leukopenia/lymphopenia & $21(58.3)$ & $9(50)$ & NS \\
\hline Thrombocytopenia & $9(25)$ & $3(16.7)$ & NS \\
\hline \multicolumn{4}{|l|}{ SLE immunologic manifestations } \\
\hline ANA & $36(100)$ & $17(94.4)$ & NS \\
\hline Anti-dsDNA & $33(91.7)$ & $12(66.7)$ & 0.047 \\
\hline Anti-Sm & $10(27.8)$ & $5(27.8)$ & NS \\
\hline Antihistone & $6(16.7)$ & $3(16.7)$ & NS \\
\hline $\mathrm{aCL}$ & $17(47.2)$ & $3(16.7)$ & 0.038 \\
\hline Anti- $\beta 2-G P$ & $10(27.8)$ & $4(22.2)$ & NS \\
\hline LAC & $6(16.7)$ & $3(16.7)$ & NS \\
\hline Low serum complement & $19(52.8)$ & $6(33.3)$ & NS \\
\hline Positive direct Coombs & $2(5.6)$ & $0(0)$ & NS \\
\hline Anti-SSA/SSB & $8(22.2)$ & $6(33.3)$ & NS \\
\hline Sicca syndrome & $9(25.0)$ & $4(22.2)$ & NS \\
\hline $\mathrm{RP}$ & $7(19.4)$ & $3(16.7)$ & NS \\
\hline RF positivity & $5(12.8)$ & $3(16.7)$ & NS \\
\hline
\end{tabular}

Values are $\mathrm{n}(\%)$ unless otherwise specified. aCL: anticardiolipin antibodies; ANA: antinuclear antibodies; anti-B2-GP: anti-B2-glycoprotein; CVD: cardiovascular disease; DM: diabetes mellitus; LAC: lupus anticoagulant; NS: not significant; PsA: psoriatic arthritis; RF: rheumatoid factor; RP: Raynaud phenomenon; SES: socioeconomic status; SLE: systemic lupus erythematosus.

and the PsA patient groups with SLE development as the outcome (Table 5), we found that female sex and PsA diagnosis were risk factors for SLE development. In this model, statin use was no longer a statistically significant risk factor for SLE development.

\section{DISCUSSION}

In our study, which is the first retrospective, large population 
Table 3. Comparison within the PsA study group between patients with and without SLE.

\begin{tabular}{|c|c|c|c|}
\hline & PsA & PsA and SLE & $P$ \\
\hline Patients, $\mathrm{n}$ & 4818 & 18 & - \\
\hline Age, yrs, mean \pm SD & $56.31 \pm 15.242$ & $54.44 \pm 15.935$ & NS \\
\hline Sex, female & $2585(53.7)$ & $18(100)$ & $<0.0001$ \\
\hline \multicolumn{4}{|l|}{ SES } \\
\hline 1 & $1544(32.2)$ & $8(44.4)$ & NS \\
\hline 2 & $1953(40.7)$ & $5(27.8)$ & \\
\hline 3 & $1303(27.1)$ & $5(27.8)$ & \\
\hline Smoker & $2044(42.5)$ & $7(38.9)$ & NS \\
\hline \multicolumn{4}{|l|}{ Ethnicity } \\
\hline Arab & $609(12.6)$ & $3(16.7)$ & NS \\
\hline Jewish & $4209(87.4)$ & $15(83.3)$ & \\
\hline \multicolumn{4}{|l|}{ Comorbidity } \\
\hline Hypertension & $2027(42.21)$ & $8(44.4)$ & NS \\
\hline $\mathrm{DM}$ & $1347(28)$ & $6(33.3)$ & NS \\
\hline Malignancy & $975(20.2)$ & $5(27.8)$ & NS \\
\hline CVA & $301(6.2)$ & $3(16.7)$ & NS \\
\hline Osteoporosis & $616(12.8)$ & $7(38.9)$ & 0.005 \\
\hline Obesity & $1847(38.3)$ & $4(22.2)$ & NS \\
\hline cDMARD & $4074(84.6)$ & $16(88.9)$ & NS \\
\hline \multicolumn{4}{|l|}{ bDMARD } \\
\hline Anti-TNF- $\alpha$ & $1774(36.8)$ & $5(27.8)$ & NS \\
\hline \multicolumn{4}{|l|}{$\begin{array}{l}\text { Possible SLE-inducing } \\
\text { medications }\end{array}$} \\
\hline Statins & $2741(56.9)$ & $9(50)$ & NS \\
\hline PPI & $3652(75.8)$ & $17(94.4)$ & NS \\
\hline Beta blockers & $474(9.8)$ & $5(27.8)$ & 0.027 \\
\hline $\mathrm{ACEi}$ & $2107(43.7)$ & $7(38.9)$ & NS \\
\hline Thiazides & $1044(21.7)$ & $4(22.2)$ & NS \\
\hline
\end{tabular}

ACEi: angiotensin-converting enzyme inhibitors; anti-TNF- $\alpha$ : anti-tumor necrosis factor- $\alpha$; bDMARD: biological disease-modifying antirheumatic drug; cDMARD: conventional disease-modifying antirheumatic drug; CVA: cerebrovascular accident; DM: diabetes mellitus; NS: not significant; PPI: proton pump inhibitor; PsA: psoriatic arthritis; SES: socioeconomic status at enrollment, as defined by the categories of 1 (low), 2 (medium), or 3 (high); SLE: systemic lupus erythematosus.

study to report on the co-existence of PSA and SLE, to our knowledge, we found a 2.3 -fold increase in the prevalence of SLE among PsA patients compared to age and sex-matched controls out of the general population $(0.37 \%$ vs $0.15 \%, P=$ 0.001 , calculated SIR $=2.81$ ). We did not find significant differences in comparing SLE disease manifestations between these 2 groups. We observed that $29.3 \%$ of PsA patients tested positive for ANA. This percentage is lower than the prevalence published in previous literature. For instance, Johnson, et al found that $47 \%$ of PsA patients had ANA positivity at a 1:40 dilution ${ }^{5}$, while Silvy, et al found 57\% ANA positivity at a 1:100 dilution and 52\% ANA positivity at a 1:160 dilution ${ }^{7}$. This was found among PsA patients where ANA testing was done as a 2-step assay with IF read by a laboratory technician followed by a separate multiplex bead assay. Therefore, differences in study populations and laboratory testing techniques might account for these differences.

In our study cohort, SLE was more prevalent in women with coexisting PsA than in men (100\% vs $53.7 \%, P<0.0001)$.
Current literature shows no difference in the occurrence of PsA among males and females in the general population. For instance, a study by Ritchlin, et al demonstrated a 1:1 male to female ratio in this regard ${ }^{15}$, as does a study by Mease, et $\mathrm{al}^{16}$. Conversely, a few other studies showed a male predominance of PsA ${ }^{17,18,19}$. Unlike PsA, SLE is known to have a much higher prevalence in females relative to males, with a 10:1 female:male ratio commonly accepted in the literature ${ }^{20}$. It is thus likely that the higher prevalence of SLE that we observed among female patients with PsA is due to the female predominance of SLE.

The literature reporting the coexistence of PsA and SLE is scarce ${ }^{9,10}$. Millns and Muller describe 27 patients with coexistent psoriasis and SLE, 10 of whom were diagnosed with SLE, 13 with discoid lupus erythematosus without systemic manifestations of SLE, and 4 with drug-induced SLE or SLE-like syndrome ${ }^{8}$. In a study by Tselios, et al, psoriasis was twice as prevalent in SLE patients compared with the general Canadian population?. A study by Zalla and Muller showed the most common type of psoriasis associated with SLE to be plaque psoriasis ${ }^{21}$. In our literature search, we found a single study showing increased prevalence of PsA among patients with SLE [diagnosed using the American College of Rheumatology (ACR) Revised Criteria from 1997] in a single tertiary, academic rheumatology specialty clinic in Syracuse, NY, with an increase in malar rash, discoid rash, photosensitivity, and arthritis in SLE patients with either coexisting psoriasis or PsA. In this study, it was also noted that antiphospholipid antibody positivity was less common in SLE patients with concurrent psoriasis or $\operatorname{PsA}^{22}$.

Possible explanations for the increase in SLE prevalence among patients with PsA include phototherapy exposure as treatment for psoriasis that may trigger SLE induction ${ }^{23}$, as could medications for PsA that increase photosensitivity such as sulfasalazine $e^{8,10,21}$. In our cohort, none of the 18 patients with concomitant SLE and PsA had phototherapy treatment prior to SLE diagnosis.

Another possible explanation for the increase in SLE prevalence among PsA patients lies in the high prevalence of comorbidities requiring medications in PsA patients relative to the general population. This higher prevalence of comorbidities among PsA which we found in our study and which was previously described in several studies ${ }^{24,25,26}$ may predispose them to drug-induced SLE or drug-induced subacute cutaneous lupus erythematosus. Of note, in our study, we only included patients fulfilling the 2012 SLICC classification criteria for SLE and did not include patients who only had skin manifestations of SLE. We found that the use of possible medications with known potential to induce SLE prior to SLE diagnosis was higher among PsA patients compared to the control group (Table 1). Using 2 separate multivariable conditional logistic regression models, we found that patients with PsA at higher risk of SLE development were patients diagnosed with PsA at an older age, those with shorter PsA disease duration, or patients on statin therapy. The association between older age of PsA onset and shorter disease duration with SLE development may lie in the higher prevalence of comorbidities found at an older age in general, and in patients with PsA in particular, necessitating a larger number and variety of 
Table 4. Assessment of risk factors for SLE development in the PsA group.

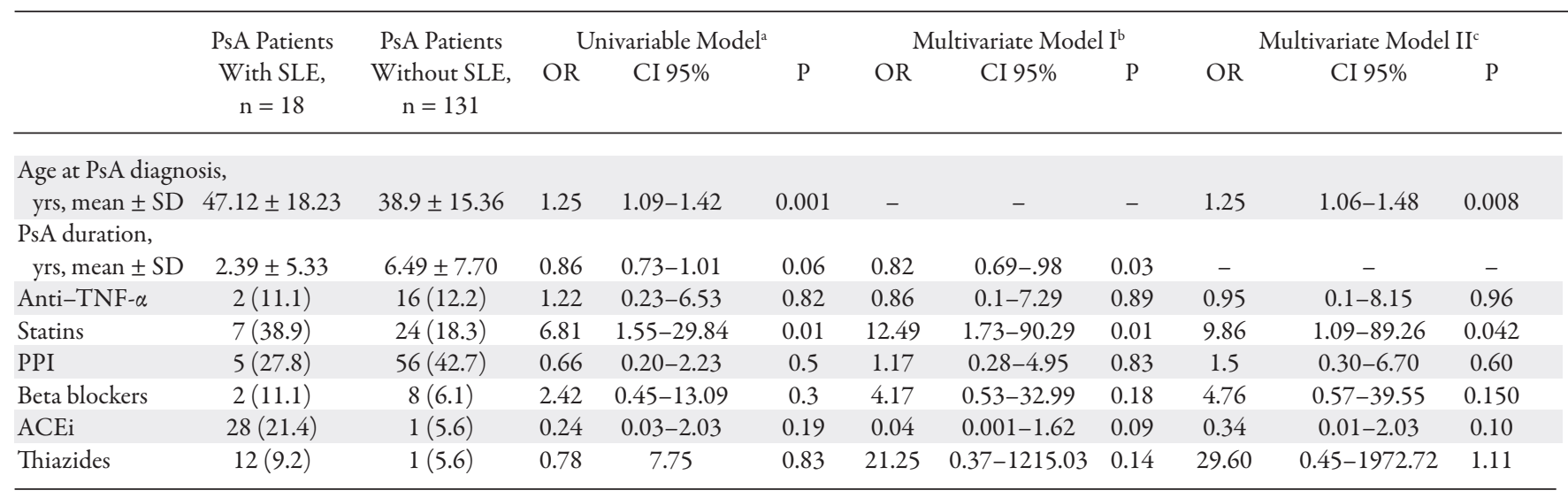

Values are n (\%) unless otherwise specified. a Logistic regression was performed separately for each item. ${ }^{\mathrm{b}}$ Multivariate Model I includes PsA duration. ${ }^{\mathrm{c}}$ Multivariate Model II includes age at PsA diagnosis. ACEi: angiotensin-converting enzyme inhibitor; anti-TNF- $\alpha$ : anti-tumor necrosis factor- $\alpha$; PPI: proton pump inhibitor; PsA: psoriatic arthritis; SLE: systemic lupus erythematosus.

Table 5. Assessment of risk factors for SLE development in the study population.

\begin{tabular}{|c|c|c|c|c|c|c|c|c|}
\hline & \multirow{2}{*}{$\begin{array}{c}\text { Population } \\
\text { With SLE }\end{array}$} & \multirow{2}{*}{$\begin{array}{c}\text { Population } \\
\text { Without SLE }\end{array}$} & \multicolumn{3}{|c|}{ Univariable Model $^{a}$} & \multicolumn{3}{|c|}{ Multivariate Model III } \\
\hline & & & OR & CI 95\% & $\mathrm{P}$ & OR & CI 95\% & $\mathrm{P}$ \\
\hline Age, yrs, mean \pm SD & $52.76 \pm 13.93$ & $56.26 \pm 15.26$ & 0.99 & $0.97-1.00$ & 0.09 & 1.01 & $0.99-1.03$ & NS \\
\hline Ethnicity, Jewish & $44(81.5)$ & $23,476(82.5)$ & 0.93 & $0.47-1.85$ & NS & & & \\
\hline PsA & $18(0.37)$ & $4818(99.6)$ & 2.50 & $1.42-4.42$ & 0.001 & 3.68 & $2.05-6.6$ & $<0.0001$ \\
\hline Anti-TNF- $\alpha$ & $2(3.7)$ & $1871(6.5)$ & 0.58 & $0.14-2.29$ & NS & & & \\
\hline Beta blockers & $3(5.6)$ & $2367(8.2)$ & 0.66 & $0.20-2.12$ & NS & & & \\
\hline $\mathrm{ACEi}$ & $8(14.8)$ & $10,742(37.1)$ & 0.30 & $0.14-0.23$ & 0.001 & 0.35 & $0.15-0.79$ & 0.012 \\
\hline Thiazides & $8(14.8)$ & $5286(18.3)$ & 0.78 & $0.37-1.65$ & NS & & & \\
\hline
\end{tabular}

Medications prescribed before SLE diagnosis. Values are n (\%) unless otherwise specified. ${ }^{a}$ Logistic regression was performed separately for each item. ACEi: angiotensin-converting enzyme inhibitor; anti-TNF- $\alpha$ : tumor necrosis factor- $\alpha$; NS: not significant; PPI: proton pump inhibitor; PsA: psoriatic arthritis; SLE: systemic lupus erythematosus.

medications in this patient population. Of these, statins in particular (irrespective of particular statin used) are identified as having a significant pharmacovigilant signal associated with SLE development in the World Health Organization Pharmacovigilance Database (VigiBase) ${ }^{27}$ with a reporting odds ratio (ROR) of lupus-like syndrome of 2.01 (95\% CI 1.61-2.51). A similar pharmacovigilant signal is also found in the French Pharmacovigilance Database for all statins except for fluvastatin, with an ROR of 1.67 (95\% CI 1.02-2.74) ${ }^{28}$. The higher incidence of hyperlipidemia among PsA patients requiring statin therapy may explain part of the increase in SLE risk among these patients ${ }^{3,24,25}$. This higher incidence of statin use among PsA patients can explain why statin use was no longer found to be a risk factor for SLE development in model III, where risk of SLE development was calculated out of the entire study population and not only in the PsA patient group as in models I and II.

Last, the implication of interleukin-23 (IL-23) and IL-17, which are known to be associated with psoriasis and PsA ${ }^{30,31}$, in SLE immunopathogenesis suggests a common underlying pathophysiology for both diseases. For instance, previous reports showed a correlation between elevated IL-17 serum levels and SLE disease activity as well as with higher anti-dsDNA antibody titers; IL-17 blockade was also shown to decrease SLE disease manifestations in animal models ${ }^{32,33}$. Fischer, et al showed elevated IL-23 serum concentrations in SLE patients with high cytokine levels associated with higher atherosclerotic plaque burden, lupus nephritis, and obesity ${ }^{34}$.

Our study's limitations consist of the retrospective design which did not capture PsA or SLE disease activity, or psoriasis subtype. In addition, as our findings are limited to the Israeli population, as diverse as this population may be, they require validation in other patient cohorts. Moreover, though our study includes data on over $50 \%$ of the Israeli population, our study is limited in our finding of a relatively small number of SLE patients within this database. Additionally, we only found a total of 131 matched control PsA patients, which fell short of 10 PsA control patients with similar follow-up time for each of the 18 patients who had PsA with coexisting SLE. 
As our risk analysis for SLE development among patients with PsA was conducted prior to the publication of the current updated European League Against Rheumatism (EULAR)/ ACR classification criteria for SLE ${ }^{35}$, the use of the current criteria relative to the 2012 SLICC criteria might have affected our findings. Notably, these criteria require ANA positivity for SLE classification, and in our study, while all of the patients in the control group had a positive ANA, 17 of 18 (94.4\%) in the PsA group with coexisting SLE had ANA positivity.

The strength of our study lies in using a large database of 4.3 million subjects with long-term follow-up for PsA. Our study is of significance because finding a positive correlation between SLE and PsA may advance our understanding of the underlying pathogenesis of both diseases and may affect treatment choices and medication development. Further, the coexistence of these diseases may in turn increase the risk of additional comorbidities such as cardiovascular and metabolic disease, depression, and/or osteoporosis, requiring closer follow-up and preventative medicine in these patients.

In conclusion, our study points to an increase in prevalence of SLE among female patients with PsA, with patients at the highest risk of SLE development being female patients with late-onset PsA, short disease duration, and especially with statin therapy. Clinicians caring for patients with PsA and SLE should be aware of the higher prevalence of comorbid conditions in this patient population. More research is needed to understand the underlying biologic pathway that may be common to SLE and PsA.

\section{REFERENCES}

1. Kasper DL, Anthony F, Stephen H, Dan L, Jameson JL, Joseph L. Harrison's principles of internal medicine. 19th ed. New York, NY: McGraw-Hill; 2015:2124-34.

2. Alinaghi F, Calov M, Kristensen LE, Gladman DD, Coates LC, Jullien D, et al. Prevalence of psoriatic arthritis in patients with psoriasis: A systematic review and meta-analysis of observational and clinical studies. J Am Acad Dermatol 2019;80:251-65.

3. Edson-Heredia E, Zhu B, Lefevre C, Wang M, Barrett A, Bushe CJ, et al. Prevalence and incidence rates of cardiovascular, autoimmune, and other diseases in patients with psoriatic or psoriatic arthritis: A retrospective study using clinical practice research datalink. J Eur Acad Dermatol Venereol 2015;29:955-63.

4. Petri M, Orbai A-M, Alarcón GS, Gordon C, Merrill JT, Fortin PR, et al. Derivation and validation of the systemic lupus international collaborating clinics classification criteria for systemic lupus erythematosus. Arthritis Rheum 2012;64:2677-86.

5. Johnson SR, Schentag CT, Gladman DD. Autoantibodies in biological agent naive patients with psoriatic arthritis. Ann Rheum Dis 2005;64:770-2.

6. Pirowska MM, Goździalska A, Lipko-Godlewska S, Obtulowicz A, Sulowicz J, Podolec K, et al. Autoimmunogenicity during anti-TNF therapy in patients with psoriasis and psoriatic arthritis. Postepy Dermatol Alergol 2015;32:250-4.

7. Silvy F, Bertin D, Bardin N, Auger I, Guzian MC, Mattei JP, et al. Antinuclear antibodies in patients with psoriatic arthritis treated or not with biologics. PLoS One 2015;10:e0134218.

8. Millns JL, Muller SA. The coexistence of psoriasis and lupus erythematosus. An analysis of 27 cases. Arch Dermatol 1980;116:658-63.
9. Tselios K, Yap KS, Pakchotanon R, Polachek A, Su J, Urowitz MB, et al. Psoriasis in systemic lupus erythematosus: a single-center experience. Clin Rheumatol 2017;36:879-84.

10. Avriel A, Zeller L, Flusser D, Abu Shakra M, Halevy S, Sukenik S. Coexistence of psoriatic arthritis and systemic lupus erythematosus. Isr Med Assoc J 2007;9:48-9.

11. Eder L, Cohen AD, Feldhamer I, Greenberg-Dotan S, Batat E, Zisman D. The epidemiology of psoriatic arthritis in Israel - a population-based study. Arthritis Res Ther 2018;20:3.

12. Zisman D, Bitterman H, Shalom G, Feldhamer I, Comanesther D, Batat E, et al. Psoriatic arthritis treatment and the risk of herpes zoster. Ann Rheum Dis 2016;75:131-5.

13. Rubin RL. Drug-induced lupus. Toxicology 2005;209:135-47.

14. Vasoo S. Drug-induced lupus: an update. Lupus 2006; 15:757-61.

15. Ritchlin CT, Colbert RA, Gladman DD. Psoriatic arthritis. N Engl J Med 2017;376:957-70.

16. Mease PJ, Karki C, Palmer JB, Etzel CJ, Kavanaugh A, Ritchlin CT, et al. Clinical characteristics, disease activity, and patient-reported outcomes in psoriatic arthritis patients with dactylitis or enthesitis: results from the Corrona psoriatic arthritis/spondyloarthritis registry. Arthritis Care Res 2017;69:1692-9.

17. Yamamoto T, Ohtsuki M, Sano S, Igarashi A, Morita A, Okuyama $\mathrm{R}$, et al. Epidemiological analysis of psoriatic arthritis patients in Japan. J Dermatol 2016;43:1193-6.

18. Nossent JC, Gran JT. Epidemiological and clinical characteristics of psoriatic arthritis in northern Norway. Scand J Rheumatol 2009;38:251-5.

19. Kumar R, Sharma A, Dogra S. Prevalence and clinical patterns of psoriatic arthritis in Indian patients with psoriasis. Indian J Dermatol Venereol Leprol 2014;80:15-23.

20. Pons-Estel GJ, Alarcón GS, Scofield L, Reinlib L, Cooper GS. Understanding the epidemiology and progression of systemic lupus erythematosus. Semin Arthritis Rheum 2010;39:257-68.

21. Zalla MJ, Muller SA. The coexistence of psoriasis with lupus erythematosus and other photosensitive disorders. Acta Derm Venereol Suppl 1996;195:1-15.

22. Bonilla E, Shadakshari A, Perl A. Association of psoriasis and psoriatic arthritis with systemic lupus erythematosus. Rheumatol Orthop Med 2016;1:1-3.

23. Eyanson S, Greist MC, Brandt KD, Skinner B. Systemic lupus erythematosus: association with psoralen-ultraviolet-a treatment of psoriasis. Arch Dermatol 1979;115:54-6.

24. Kibari A, Cohen AD, Gazitt T, Bitterman H, Lavi I, Feldhamer I, et al. Cardiac and cardiovascular morbidities in patients with psoriatic arthritis: a population-based case control study. Clin Rheumatol 2019;38:2069-75.

25. Ogdie A, Schwartzman S, Eder L, Maharaj AB, Zisman D, Raychaudhuri SP, et al. Comprehensive treatment of psoriatic arthritis: managing comorbidities and extraarticular manifestations. J Rheumatol 2014;41:2315-22.

26. Polachek A, Touma Z, Anderson M, Eder L. Risk of cardiovascular morbidity in patients with psoriatic arthritis: a meta-analysis of observational studies. Arthritis Care Res 2017;69:67-74.

27. de Jong HJ, Tervaert JW, Saldi SR, Vandebriel RJ, Souverein PC, Meyboom RH, et al. Association between statin use and lupuslike syndrome using spontaneous reports. Semin Arthritis Rheum 2011;41:373-81.

28. Moulis G, Bene J, Sommet A, Sailler L, Lapeyre-Mestre M, Montastruc JL, et al; French Association of PharmacoVigilance Centres. Statin-induced lupus: a case/non-case study in a nationwide pharmacovigilance database. Lupus 2012;21:885-9.

29. Vaz JL, Andrade CA, Pereira AC, Martins MdF, Levy RA. Systematic review of infliximab-induced autoantibodies and systemic lupus erythematosus. Rev Bras Reumatol 2013;53:358-64. 
30. Murdaca G, Colombo BM, Puppo F. The role of Th17 lymphocytes in the autoimmune and chronic inflammatory diseases. Intern Emerg Med 2011;6:487-95.

31. Tabarkiewicz J, Pogoda K, Karczmarczyk A, Pozarowski P, Giannopoulos K. The role of IL-17 and Th17 lymphocytes in autoimmune diseases. Arch Immunol Ther Exp 2015;63:435-49.

32. Nalbandian A, Crispín JC, Tsokos GC. Interleukin-17 and systemic lupus erythematosus: current concepts. Clin Exp Immunol 2009;157:209-15.

33. Leng R-X, Pan HF, Chen GM, Wang C, Qin WZ, Chen LL, et al. IL-23: a promising therapeutic target for systemic lupus erythematosus. Arch Med Res 2010;41:221-5.
34. Fischer K, Przepiera-Będzak H, Sawicki M, Walecka A, Brzosko I, Brzosko M. Serum interleukin-23 in polish patients with systemic lupus erythematosus: association with lupus nephritis, obesity, and peripheral vascular disease. Mediators Inflamm 2017;2017:9401432.

35. Aringer M, Costenbader K, Daikh D, Brinks R, Mosca M, Ramsey-Goldman R, et al. 2019 European League Against Rheumatism/American College of Rheumatology Classification Criteria for Systemic Lupus Erythematosus. Arthritis Rheumatol 2019;71:1400-12. 